\title{
Solvability of Nonlinear Langevin Equation Involving Two Fractional Orders with Dirichlet Boundary Conditions
}

\author{
Bashir Ahmad ${ }^{1}$ and Juan J. Nieto ${ }^{2}$ \\ ${ }^{1}$ Department of Mathematics, Faculty of Science, King Abdulaziz University, P.O. Box 80203, \\ Jeddah 21589, Saudi Arabia \\ ${ }^{2}$ Departamento de Análisis Matemático, Facultad de Matemáticas, Universidad de Santiago de Compostela, \\ 15782 Santiago de Compostela, Spain
}

Correspondence should be addressed to Bashir Ahmad, bashir_qau@yahoo.com

Received 8 August 2009; Accepted 14 November 2009

Academic Editor: Nikolai Leonenko

Copyright (C) 2010 B. Ahmad and J. J. Nieto. This is an open access article distributed under the Creative Commons Attribution License, which permits unrestricted use, distribution, and reproduction in any medium, provided the original work is properly cited.

\begin{abstract}
We study a Dirichlet boundary value problem for Langevin equation involving two fractional orders. Langevin equation has been widely used to describe the evolution of physical phenomena in fluctuating environments. However, ordinary Langevin equation does not provide the correct description of the dynamics for systems in complex media. In order to overcome this problem and describe dynamical processes in a fractal medium, numerous generalizations of Langevin equation have been proposed. One such generalization replaces the ordinary derivative by a fractional derivative in the Langevin equation. This gives rise to the fractional Langevin equation with a single index. Recently, a new type of Langevin equation with two different fractional orders has been introduced which provides a more flexible model for fractal processes as compared with the usual one characterized by a single index. The contraction mapping principle and Krasnoselskii's fixed point theorem are applied to prove the existence of solutions of the problem in a Banach space.
\end{abstract}

\section{Introduction}

Fractional differential equations have recently gained much importance and attention. The study of fractional differential equations ranges from the theoretical aspects of existence and uniqueness of solutions to the analytic and numerical methods for finding solutions. Fractional differential equations appear naturally in a number of fields such as physics, polymer rheology, regular variation in thermodynamics, biophysics, blood flow phenomena, aerodynamics, electrodynamics of complex medium, viscoelasticity, 
Bodes analysis of feedback amplifiers, capacitor theory, electrical circuits, electronanalytical chemistry, biology, control theory, fitting of experimental data, etc. An excellent account in the study of fractional differential equations can be found in [1-3]. For more details and examples, see [4-13] and the references therein. Some new and recent aspects on fractional calculus can be seen in [14-16]. In [15], it was shown that fractional Nambu systems can be proposed as a generalization of fractional Hamiltonian systems.

Langevin equation is widely used to describe the evolution of physical phenomena in fluctuating environments [17]. However, for the systems in complex media, ordinary Langevin equation does not provide the correct description of the dynamics. One of the possible generalizations of Langevin equation is to replace the ordinary derivative by a fractional derivative in it. This gives rise to fractional Langevin equation, see for instance $[18,19]$ and the references therein. In [18], the authors studied a new type of Langevin equation with two different fractional orders. The solution to this new version of fractional Langevin equation gives a fractional Gaussian process parameterized by two indices, which provides a more flexible model for fractal processes as compared with the usual one characterized by a single index. In [19], the fractional oscillator process with two indices was discussed.

In this paper, we study a Dirichlet boundary value problem of Langevin equation with two different fractional orders. This work is motivated by recent work of Lim et al. $[18,19]$. Precisely, we consider the problem

$$
\begin{gathered}
{ }^{c} D^{\beta}\left({ }^{c} D^{\alpha}+\lambda\right) x(t)=f(t, x(t)), \quad 0<t<1,0<\alpha, \beta \leq 1, \\
x(0)=\gamma_{1}, \quad x(1)=\gamma_{2},
\end{gathered}
$$

where ${ }^{c} D$ is the Caputo fractional derivative, $f:[0,1] \times X \rightarrow X, \lambda$ is a real number and $\gamma_{1}, \gamma_{2} \in X$. Here, $(X,\|\cdot\|)$ is a Banach space and $C=C([0,1], X)$ denotes the Banach space of all continuous functions from $[0,1] \rightarrow X$ endowed with a topology of uniform convergence with norm defined by $\|x\|=\sup \{|x(t)|, t \in[0,1]\}$.

In Section 1, we prove a new result for linear differential equations involving two fractional orders. Section 2 deals with the theory of nonlinear differential equations with two fractional orders. We first use the contraction mapping principle to prove the existence and uniqueness of the solution of problem (1.1) in a Banach space. We then employ Krasnoselskii's fixed point theorem to establish another new existence result for problem (1.1). We also give an example for the illustration of the theory established in this paper.

A function $x \in \mathcal{C}$ with its Caputo derivative of fractional order existing on $(0,1)$ is a solution of (1.1) if it satisfies (1.1).

Relative to (1.1), we now introduce the following linear problem:

$$
\begin{array}{rlrl}
{ }^{c} D^{\beta}\left({ }^{c} D^{\alpha}+\lambda\right) x(t) & =\sigma(t), & & 0<t<1,0<\alpha, \beta \leq 1, \\
x(0) & =\gamma_{1}, \quad & x(1)=\gamma_{2},
\end{array}
$$

where $\sigma \in C[0,1]$. 
Lemma 1.1. The unique solution of the boundary value problem (1.2) is given by

$$
\begin{aligned}
x(t)= & \int_{0}^{t} \frac{(t-u)^{\alpha-1}}{\Gamma(\alpha)}\left(\int_{0}^{u} \frac{(u-s)^{\beta-1}}{\Gamma(\beta)} \sigma(s) d s-\lambda x(u)\right) d u \\
& -t^{\alpha}\left[\int_{0}^{1} \frac{(1-u)^{\alpha-1}}{\Gamma(\alpha)}\left(\int_{0}^{u} \frac{(u-s)^{\beta-1}}{\Gamma(\beta)} \sigma(s) d s-\lambda x(u)\right) d u\right]+\left(\gamma_{2}-\gamma_{1}\right) t^{\alpha}+\gamma_{1} .
\end{aligned}
$$

Proof. As argued in [2, Section 5.4], the general solution of

$$
{ }^{c} D^{\beta}\left({ }^{c} D^{\alpha}+\lambda\right) x(t)=\sigma(t)
$$

can be written as

$$
x(t)=\int_{0}^{t} \frac{(t-u)^{\alpha-1}}{\Gamma(\alpha)}\left(\int_{0}^{u} \frac{(u-s)^{\beta-1}}{\Gamma(\beta)} \sigma(s) d s-\lambda x(u)\right) d u-\frac{c_{0}}{\Gamma(\alpha+1)} t^{\alpha}-c_{1} .
$$

Using the boundary conditions for (1.2), we find that

$$
c_{1}=-\gamma_{1}, \quad \frac{c_{0}}{\Gamma(\alpha+1)}=\int_{0}^{1} \frac{(1-u)^{\alpha-1}}{\Gamma(\alpha)}\left(\int_{0}^{u} \frac{(u-s)^{\beta-1}}{\Gamma(\beta)} \sigma(s) d s-\lambda x(u)\right) d u-\gamma_{2}+\gamma_{1} .
$$

Substituting (1.6) in (1.5), we obtain the solution given by (1.3). This completes the proof.

Now, we state a known result due to Krasnoselskii (see [20]) which is needed to prove the existence of at least one solution of (1.1). 
Theorem 1.2. Let $M$ be a closed convex and nonempty subset of a Banach space X. Let $A, B$ be the operators such that (i) $A x+B y \in M$ whenever $x, y \in M$; (ii) $A$ is compact and continuous; (iii) $B$ is a contraction mapping. Then there exists $z \in M$ such that $z=A z+B z$.

\section{Existence of Solutions}

Theorem 2.1. Let $f:[0,1] \times X \rightarrow X$ be a jointly continuous function satisfying the condition

$$
|f(t, x)-f(t, y)| \leq L|x-y|, \quad \forall t \in[0,1], x, y \in X
$$

Then the boundary value problem (1.1) has a unique solution provided $\Lambda<1$, where

$$
\Lambda=\frac{2 L}{\Gamma(\alpha+\beta+1)}+\frac{2|\lambda|}{\Gamma(\alpha+1)}
$$

Proof. Define $\digamma: \mathcal{C} \rightarrow \mathcal{C}$ by

$$
\begin{aligned}
(\digamma x)(t)= & \int_{0}^{t} \frac{(t-u)^{\alpha-1}}{\Gamma(\alpha)}\left(\int_{0}^{u} \frac{(u-s)^{\beta-1}}{\Gamma(\beta)} f(s, x(s)) d s-\lambda x(u)\right) d u \\
& -t^{\alpha}\left[\int_{0}^{1} \frac{(1-u)^{\alpha-1}}{\Gamma(\alpha)}\left(\int_{0}^{u} \frac{(u-s)^{\beta-1}}{\Gamma(\beta)} f(s, x(s)) d s-\lambda x(u)\right) d u\right] \\
& +\left(\gamma_{2}-\gamma_{1}\right) t^{\alpha}+\gamma_{1}, \quad t \in[0,1] .
\end{aligned}
$$

Let us set $\sup _{t \in[0,1]}|f(t, 0)|=M$ and choose

$$
r \geq \frac{1}{1-\delta}\left(\frac{2 M}{\Gamma(\alpha+\beta+1)}+\left(\left|\gamma_{2}\right|+2\left|\gamma_{1}\right|\right)\right)
$$


where $\delta$ is such that $\Lambda \leq \delta<1$. Now we show that $\digamma B_{r} \subset B_{r}$, where $B_{r}=\{x \in \mathcal{C}:\|x\| \leq r\}$. For $x \in B_{r}$, we have

$\|(\digamma x)(t)\|$

$$
\begin{aligned}
& =\sup _{t \in[0,1]} \mid \int_{0}^{t} \frac{(t-u)^{\alpha-1}}{\Gamma(\alpha)}\left(\int_{0}^{u} \frac{(u-s)^{\beta-1}}{\Gamma(\beta)} f(s, x(s)) d s-\lambda x(u)\right) d u \\
& -t^{\alpha}\left[\int_{0}^{1} \frac{(1-u)^{\alpha-1}}{\Gamma(\alpha)}\left(\int_{0}^{u} \frac{(u-s)^{\beta-1}}{\Gamma(\beta)} f(s, x(s)) d s-\lambda x(u)\right) d u\right]+\left(\gamma_{2}-\gamma_{1}\right) t^{\alpha}+\gamma_{1} \mid \\
& \leq \sup _{t \in[0,1]}\left(\int_{0}^{t} \frac{(t-u)^{\alpha-1}}{\Gamma(\alpha)}\left(\int_{0}^{u} \frac{(u-s)^{\beta-1}}{\Gamma(\beta)}(|f(s, x(s))-f(s, 0)|+|f(s, 0)|) d s+|\lambda x(u)|\right) d u\right. \\
& +t^{\alpha}\left[\int _ { 0 } ^ { 1 } \frac { ( 1 - u ) ^ { \alpha - 1 } } { \Gamma ( \alpha ) } \left(\int_{0}^{u} \frac{(u-s)^{\beta-1}}{\Gamma(\beta)}(|f(s, x(s))-f(s, 0)|+|f(s, 0)|) d s\right.\right. \\
& \left.+|\lambda x(u)|) d u]+\left(\left|\gamma_{2}\right|+\left|\gamma_{1}\right|\right) t^{\alpha}+\left|\gamma_{1}\right|\right) \\
& \leq \sup _{t \in[0,1]}\left(\int_{0}^{t} \frac{(t-u)^{\alpha-1}}{\Gamma(\alpha)}\left(\int_{0}^{u} \frac{(u-s)^{\beta-1}}{\Gamma(\beta)}(L|x(s)|+|f(s, 0)|) d s+|\lambda x(u)|\right) d u\right. \\
& +t^{\alpha}\left[\int_{0}^{1} \frac{(1-u)^{\alpha-1}}{\Gamma(\alpha)}\left(\int_{0}^{u} \frac{(u-s)^{\beta-1}}{\Gamma(\beta)}(L|x(s)|+|f(s, 0)|) d s+|\lambda x(u)|\right) d u\right] \\
& \left.+\left(\left|\gamma_{2}\right|+\left|\gamma_{1}\right|\right) t^{\alpha}+\left|\gamma_{1}\right|\right) \\
& \leq \sup _{t \in[0,1]}\left(\int_{0}^{t} \frac{(t-u)^{\alpha-1}}{\Gamma(\alpha)}\left(\int_{0}^{u} \frac{(u-s)^{\beta-1}}{\Gamma(\beta)} d s(L|x(u)|+|f(u, 0)|)+|\lambda x(u)|\right) d u\right. \\
& +t^{\alpha}\left[\int_{0}^{1} \frac{(1-u)^{\alpha-1}}{\Gamma(\alpha)}\left(\int_{0}^{u} \frac{(u-s)^{\beta-1}}{\Gamma(\beta)} d s(L|x(u)|+|f(u, 0)|)+|\lambda x(u)|\right) d u\right] \\
& \left.+\left(\left|r_{2}\right|+\left|\gamma_{1}\right|\right) t^{\alpha}+\left|r_{1}\right|\right) \\
& \leq \sup _{t \in[0,1]} \int_{0}^{t} \frac{(t-u)^{\alpha-1}}{\Gamma(\alpha)} \int_{0}^{u} \frac{(u-s)^{\beta-1}}{\Gamma(\beta)} d s d u(L\|x\|+|M|)+\sup _{t \in[0,1]} \int_{0}^{t} \frac{(t-u)^{\alpha-1}}{\Gamma(\alpha)} d u|\lambda|\|x\| \\
& +\int_{0}^{1} \frac{(1-u)^{\alpha-1}}{\Gamma(\alpha)} \int_{0}^{u} \frac{(u-s)^{\beta-1}}{\Gamma(\beta)} d s d u(L\|x\|+M)+\int_{0}^{1} \frac{(1-u)^{\alpha-1}}{\Gamma(\alpha)} d u|\lambda||| x \|+\left|\gamma_{2}\right|+2\left|\gamma_{1}\right|
\end{aligned}
$$




$$
\begin{aligned}
& \leq 2(L r+M) \int_{0}^{1} \frac{(1-u)^{\alpha-1}}{\Gamma(\alpha)} \int_{0}^{u} \frac{(u-s)^{\beta-1}}{\Gamma(\beta)} d s d u \\
& \quad+2|\lambda| r \int_{0}^{1} \frac{(1-u)^{\alpha-1}}{\Gamma(\alpha)} d u+\left(\left|\gamma_{2}\right|+2\left|\gamma_{1}\right|\right) \\
& =\frac{2(L r+M)}{\Gamma(\alpha) \Gamma(\beta+1)} \int_{0}^{1}(1-u)^{\alpha-1} u^{\beta} d u+\frac{2|\lambda| r}{\Gamma(\alpha+1)}+\left(\left|\gamma_{2}\right|+2\left|\gamma_{1}\right|\right) .
\end{aligned}
$$

Using (2.2), (2.4), and the relation for Beta function $B(\cdot, \cdot)$ :

$$
B(\beta+1, \alpha)=\int_{0}^{1}(1-u)^{\alpha-1} u^{\beta} d u=\frac{\Gamma(\alpha) \Gamma(\beta+1)}{\Gamma(\alpha+\beta+1)}
$$

we find that

$$
\|(\digamma x)(t)\| \leq(\Lambda+1-\delta) r \leq r .
$$

Now, for $x, y \in \mathcal{C}$ and for each $t \in[0,1]$, we obtain

$$
\begin{aligned}
& \|(\digamma x)(t)-(\digamma y)(t)\| \\
& =\sup _{t \in[0,1]}|(\digamma x)(t)-(\digamma y)(t)| \\
& \leq \sup _{t \in[0,1]}\left(\int_{0}^{t} \frac{(t-u)^{\alpha-1}}{\Gamma(\alpha)}\left(\int_{0}^{u} \frac{(u-s)^{\beta-1}}{\Gamma(\beta)}|f(s, x(s))-f(s, y(s))| d s\right) d u\right. \\
& +|\lambda| \int_{0}^{t} \frac{(t-u)^{\alpha-1}}{\Gamma(\alpha)}(|x(u)-y(s)|) d u \\
& \quad+t^{\alpha}\left[\int_{0}^{1} \frac{(1-u)^{\alpha-1}}{\Gamma(\alpha)}\left(\int_{0}^{u} \frac{(u-s)^{\beta-1}}{\Gamma(\beta)}|f(s, x(s))-f(s, y(s))| d s\right) d u\right. \\
& \left.\left.+|\lambda| \int_{0}^{1} \frac{(1-u)^{\alpha-1}}{\Gamma(\alpha)}|x(u)-y(u)| d u\right]\right)
\end{aligned}
$$


International Journal of Differential Equations

$$
\begin{aligned}
& \leq \sup _{t \in[0,1]}\left(L \int_{0}^{t} \frac{(t-u)^{\alpha-1}}{\Gamma(\alpha)} \int_{0}^{u} \frac{(u-s)^{\beta-1}}{\Gamma(\beta)} d s d u|x(t)-y(t)|\right. \\
& \left.\quad+|\lambda| \int_{0}^{t} \frac{(t-u)^{\alpha-1}}{\Gamma(\alpha)} d u|x(t)-y(t)|\right) \\
& \quad+L \int_{0}^{1} \frac{(1-u)^{\alpha-1}}{\Gamma(\alpha)} \int_{0}^{u} \frac{(u-s)^{\beta-1}}{\Gamma(\beta)} d s d u\|x-y\| \\
& \quad+|\lambda| \int_{0}^{1} \frac{(1-u)^{\alpha-1}}{\Gamma(\alpha)} d u\|x-y\| \\
& \leq\|x-y\|\left[2 L \int_{0}^{1} \frac{(1-u)^{\alpha-1}}{\Gamma(\alpha)} \int_{0}^{u} \frac{(u-s)^{\beta-1}}{\Gamma(\beta)} d s d u+2|\lambda| \int_{0}^{1} \frac{(1-u)^{\alpha-1}}{\Gamma(\alpha)} d u\right] \\
& =\Lambda\|x-y\|,
\end{aligned}
$$

where

$$
\Lambda=\frac{2 L}{\Gamma(\alpha+\beta+1)}+\frac{2|\lambda|}{\Gamma(\alpha+1)}
$$

which depends only on the parameters involved in the problem. As $\Lambda<1$, then $\digamma$ is a contraction. Thus, the conclusion of the theorem follows by the contraction mapping principle. This completes the proof.

Theorem 2.2. Assume that $f:[0,1] \times X \rightarrow X$ is a jointly continuous function and maps bounded subsets of $[0,1] \times X$ into relatively compact subsets of $X$. Furthermore, assume that

$\left(\mathrm{H}_{1}\right)|f(t, x)-f(t, y)| \leq L|x-y|$, for all $t \in[0,1], x, y \in X$;

$\left(\mathrm{H}_{2}\right)|f(t, x)| \leq \mu(t)$, for all $(t, x) \in[0,1] \times X$, and $\mu \in L^{1}\left([0,1], R^{+}\right)$.

If

$$
\left(\frac{L}{\Gamma(\alpha+\beta+1)}+\frac{|\lambda|}{\Gamma(\alpha+1)}\right)<1,
$$

then the boundary value problem (1.1) has at least one solution on $[0,1]$.

Proof. Let us fix

$$
r \geq\left(\frac{2\|\mu\|_{L^{1}} / \Gamma(\alpha+\beta+1)+\left|\gamma_{2}\right|+2\left|\gamma_{1}\right|}{1-2|\lambda| / \Gamma(\alpha+1)}\right)
$$


and consider $B_{r}=\{x \in \mathcal{C}:\|x\| \leq r\}$. We define the operators $\Phi$ and $\Psi$ on $B_{r}$ as

$$
\begin{aligned}
(\Phi x)(t)= & \int_{0}^{t} \frac{(t-u)^{\alpha-1}}{\Gamma(\alpha)}\left(\int_{0}^{u} \frac{(u-s)^{\beta-1}}{\Gamma(\beta)} f(s, x(s)) d s-\lambda x(u)\right) d u \\
(\Psi x)(t)= & -t^{\alpha}\left[\int_{0}^{1} \frac{(1-u)^{\alpha-1}}{\Gamma(\alpha)}\left(\int_{0}^{u} \frac{(u-s)^{\beta-1}}{\Gamma(\beta)} f(s, x(s)) d s-\lambda x(u)\right) d u\right] \\
& +\left(\gamma_{2}-\gamma_{1}\right) t^{\alpha}+\gamma_{1} .
\end{aligned}
$$

For $x, y \in B_{r}$, we find that

$$
\|\Phi x+\Psi y\| \leq\left(\frac{2\|\mu\|_{L^{1}}}{\Gamma(\alpha+\beta+1)}+\frac{2|\lambda| r}{\Gamma(\alpha+1)}+\left|\gamma_{2}\right|+2\left|\gamma_{1}\right|\right) \leq r
$$

Thus, $\Phi x+\Psi y \in B_{r}$. From the assumption

$$
\left(\frac{L}{\Gamma(\alpha+\beta+1)}+\frac{|\lambda|}{\Gamma(\alpha+1)}\right)<1
$$

it follows that $\Psi$ is a contraction mapping. The continuity of $f$ implies that the operator $\Phi$ is continuous. Also, $\Phi$ is uniformly bounded on $B_{r}$ as

$$
\|\Phi x\| \leq \frac{\|\mu\|_{L^{1}}}{\Gamma(\alpha+\beta+1)}+\frac{|\lambda| r}{\Gamma(\alpha+1)} .
$$

Now we prove the compactness of the operator $\Phi$. Setting $\Omega=[0,1] \times B_{r}$, we define $\sup _{(t, x) \in \Omega}|f(t, x)|=\bar{f}$, and consequently we have

$$
\begin{aligned}
\left\|(\Phi x)\left(t_{1}\right)-(\Phi x)\left(t_{2}\right)\right\|= & \| \int_{0}^{t_{1}} \frac{\left(t_{1}-u\right)^{\alpha-1}}{\Gamma(\alpha)}\left(\int_{0}^{u} \frac{(u-s)^{\beta-1}}{\Gamma(\beta)} f(s, x(s)) d s-\lambda x(u)\right) d u \\
& -\int_{0}^{t_{2}} \frac{\left(t_{2}-u\right)^{\alpha-1}}{\Gamma(\alpha)}\left(\int_{0}^{u} \frac{(u-s)^{\beta-1}}{\Gamma(\beta)} f(s, x(s)) d s-\lambda x(u)\right) d u \| \\
\leq & \frac{\bar{f}}{\Gamma(\alpha+\beta+1)}\left|t_{1}^{\alpha+\beta}-t_{2}^{\alpha+\beta}\right|+\frac{|\lambda| r}{\Gamma(\alpha+1)}\left|t_{1}^{\alpha}-t_{2}^{\alpha}\right|
\end{aligned}
$$

which is independent of $x$. Thus, $\Phi$ is equicontinuous. Using the fact that $f$ maps bounded subsets into relatively compact subsets, we have that $\Phi(\mathcal{A})(t)$ is relatively compact in $X$ for every $t$, where $\mathcal{A}$ is a bounded subset of $\mathcal{C}$. So $\Phi$ is relatively compact on $B_{r}$. Hence, by the Arzela Ascoli theorem, $\Phi$ is compact on $B_{r}$. Thus all the assumptions of Theorem 1.2 are satisfied and the conclusion of Theorem 1.2 implies that the boundary value problem (1.1) has at least one solution on $[0,1]$. This completes the proof. 
Example 2.3. Consider the boundary value problem

$$
\begin{gathered}
{ }^{c} D^{1 / 4}\left({ }^{c} D^{1 / 2}+\frac{1}{4}\right) x(t)=\frac{1}{(t+3)^{2}} \frac{|x|}{1+|x|}, \quad 0<t<1, \\
x(0)=\gamma_{1}, \quad x(1)=\gamma_{2} .
\end{gathered}
$$

Here, $f(t, x)=\left(1 /(t+3)^{2}\right)(|x| /(1+|x|)), \alpha=1 / 2, \beta=1 / 4$ and $\lambda=1 / 4$. Clearly $\mid f(t, x)-$ $f(t, y)|\leq(1 / 9)| x-y \mid$ with $L=1 / 9$. Further,

$$
\Lambda=\frac{8}{27 \Gamma(3 / 4)}+\frac{1}{\sqrt{\pi}}<1
$$

Thus, by Theorem 2.1, the boundary value problem (2.17) has a unique solution on $[0,1]$.

\section{Conclusions}

The existence of solutions for a Dirichlet boundary value problem involving Langevin equation with two different fractional orders has been discussed. We apply the concepts of fractional calculus together with fixed point theorems to establish the existence results. First of all, we find the unique solution for a linear Dirichlet boundary value problem involving Langevin equation with two different fractional orders, which in fact provides the platform to prove the existence of solutions for the associated nonlinear fractional Langevin equation with two different orders. Our approach is simple and is applicable to a variety of real world problems.

\section{Acknowledgments}

The research of J. J. Nieto has been supported by Ministerio de Educacion y Ciencia and FEDER, project MTM2007-61724, and by Xunta de Galicia and FEDER, project PGIDIT06PXIB207023PR.

\section{References}

[1] A. A. Kilbas, H. M. Srivastava, and J. J. Trujillo, Theory and Applications of Fractional Differential Equations, vol. 204 of North-Holland Mathematics Studies, Elsevier Science B. V., Amsterdam, The Netherlands, 2006.

[2] V. Lakshmikantham, S. Leela, and J. Vasundhara Devi, Theory of Fractional Dynamic Systems, Cambridge Academic, Cambridge, UK, 2009.

[3] I. Podlubny, Fractional Differential Equations, Academic Press, San Diego, Calif., USA, 1999.

[4] B. Ahmad and J. J. Nieto, "Existence results for nonlinear boundary value problems of fractional integrodifferential equations with integral boundary conditions," Boundary Value Problems, vol. 2009, Article ID 708576, 11 pages, 2009.

[5] B. Ahmad and J. J. Nieto, "Existence of solutions for nonlocal boundary value problems of higherorder nonlinear fractional differential equations," Abstract and Applied Analysis, vol. 2009, Article ID 494720, 9 pages, 2009. 
[6] B. Ahmad and J. J. Nieto, "Existence results for a coupled system of nonlinear fractional differential equations with three-point boundary conditions," Computers \& Mathematics with Applications, vol. 58, no. 9, pp. 1838-1843, 2009.

[7] B. Ahmad and J. J. Nieto, "Existence of solutions for anti-periodic boundary value problems involving fractional differential equations via Leray-Schauder degree theory," to appear in Topological Methods in Nonlinear Analysis.

[8] B. Ahmad, "Existence of solutions for irregular boundary value problems involving nonlinear fractional differential equations," Applied Mathematics Letters, 2009.

[9] B. Ahmad and J. J. Nieto, "Existence of solutions for impulsive anti-periodic boundary value problems of fractional order," to appear in Taiwanese Journal of Mathematics.

[10] Y.-K. Chang and J. J. Nieto, "Some new existence results for fractional differential inclusions with boundary conditions," Mathematical and Computer Modelling, vol. 49, no. 3-4, pp. 605-609, 2009.

[11] V. Daftardar-Gejji and S. Bhalekar, "Boundary value problems for multi-term fractional differential equations," Journal of Mathematical Analysis and Applications, vol. 345, no. 2, pp. 754-765, 2008.

[12] R. Hilfer, Ed., Applications of Fractional Calculus in Physics, World Scientific, River Edge, NJ, USA, 2000.

[13] S. Z. Rida, H. M. El-Sherbiny, and A. A. M. Arafa, "On the solution of the fractional nonlinear Schrödinger equation," Physics Letters A, vol. 372, no. 5, pp. 553-558, 2008.

[14] A. Arara, M. Benchohra, N. Hamidi, and J. J. Nieto, "Fractional order differential equations on an unbounded domain," Nonlinear Analysis, vol. 72, pp. 580-586, 2010.

[15] D. Baleanu, A. K. Golmankhaneh, and A. K. Golmankhaneh, "Fractional Nambu mechanics," International Journal of Theoretical Physics, vol. 48, no. 4, pp. 1044-1052, 2009.

[16] M. R. Ubriaco, "Entropies based on fractional calculus," Physics Letters A, vol. 373, no. 30, pp. 25162519, 2009.

[17] W. T. Coffey, Yu. P. Kalmykov, and J. T. Waldron, The Langevin Equation: With Applications to Stochastic Problems in Physics, Chemistry and Electrical Engineering, vol. 14 of World Scientific Series in Contemporary Chemical Physics, World Scientific, River Edge, NJ, USA, 2nd edition, 2004.

[18] S. C. Lim, M. Li, and L. P. Teo, "Langevin equation with two fractional orders," Physics Letters A, vol. 372, no. 42, pp. 6309-6320, 2008.

[19] S. C. Lim and L. P. Teo, "The fractional oscillator process with two indices," Journal of Physics A, vol. 42, no. 6, Article ID 065208, 34 pages, 2009

[20] D. R. Smart, Fixed Point Theorems, Cambridge University Press, Cambridge, UK, 1980. 


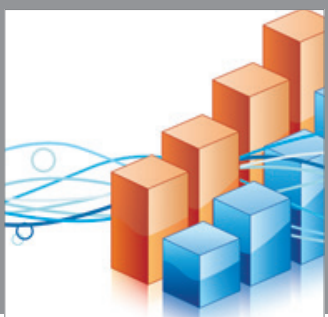

Advances in

Operations Research

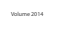

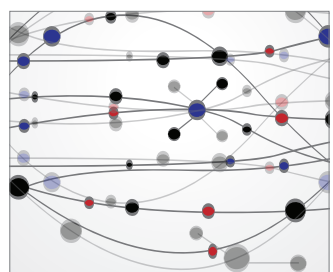

\section{The Scientific} World Journal
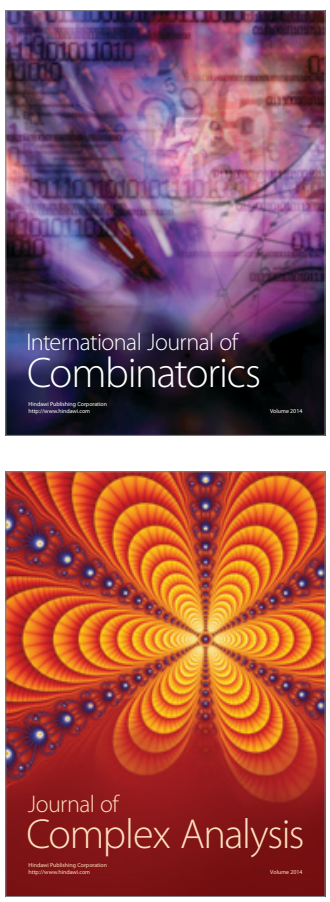

International Journal of

Mathematics and

Mathematical

Sciences
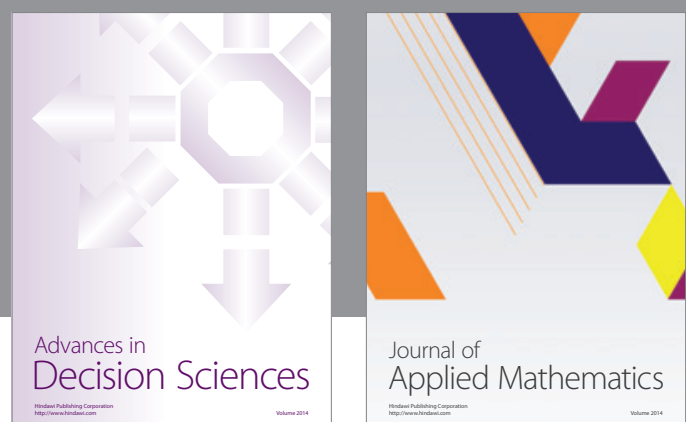

Journal of

Applied Mathematics
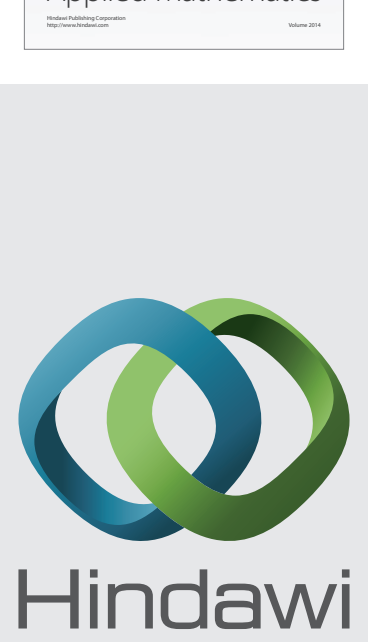

Submit your manuscripts at http://www.hindawi.com
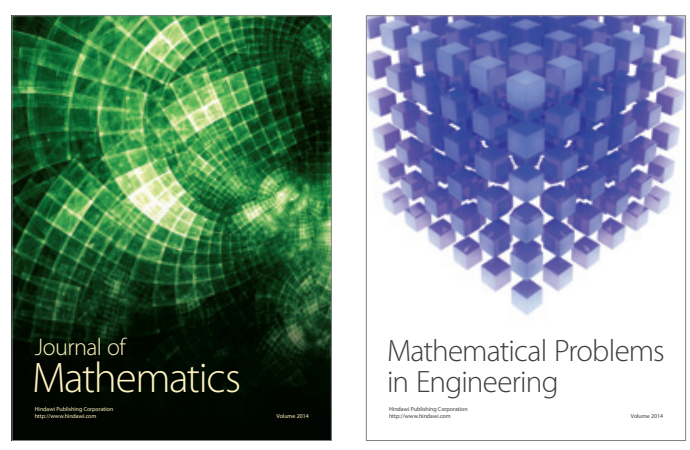

Mathematical Problems in Engineering
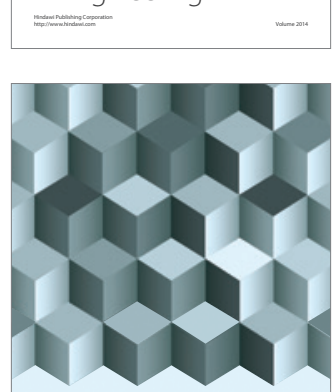

Journal of

Function Spaces
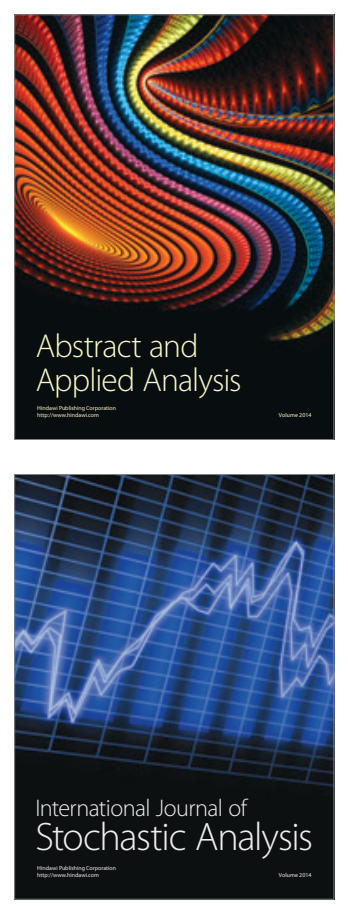

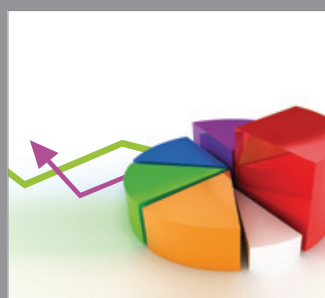

ournal of

Probability and Statistics

Promensencen
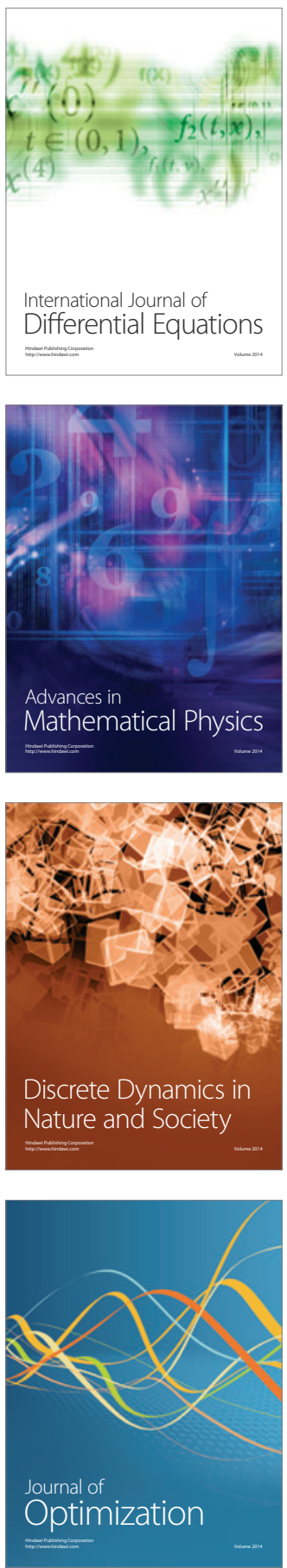